\title{
MORE RESULTS IN POLYCHROMATIC RAMSEY THEORY
}

\author{
URI ABRAHAM AND JAMES CUMMINGS
}

\begin{abstract}
We study polychromatic Ramsey theory with a focus on colourings of $\left[\omega_{2}\right]^{2}$. We show that in the absence of $\mathrm{GCH}$ there is a wide range of possibilities. In particular each of the following is consistent relative to the consistency of ZFC:
\end{abstract}

(1) $2^{\omega}=\omega_{2}$ and $\omega_{2} \rightarrow$ poly $(\alpha)_{\aleph_{0}-\text { bdd }}^{2}$ for every $\alpha<\omega_{2}$.

(2) $2^{\omega}=\omega_{2}$ and $\omega_{2} \nrightarrow^{\text {poly }}\left(\omega_{1}\right)_{2-\text { bdd }}^{2}$

\section{IntRoduction}

Polychromatic Ramsey theory (also sometimes known as rainbow Ramsey theory or sub-Ramsey theory) is dual in a certain sense to classical Ramsey theory. In classical Ramsey theory we are concerned with the existence of homogeneous or monochromatic sets for colourings of the set $[X]^{n}$ of unordered $n$-tuples from a set $X$, where a set $Y \subseteq X$ is homogeneous when $f \uparrow[Y]^{n}$ is constant. In polychromatic Ramsey theory we go to the other extreme and study sets $Y \subseteq X$ such that $f \uparrow[Y]^{n}$ is 1-1. Such sets are called polychromatic or rainbow.

In classical Ramsey theory we usually study colourings in a fixed number of colours. A natural hypothesis for the polychromatic theory is that each colour occurs a bounded number of times. If $\kappa$ is a cardinal and $f:[X]^{n} \rightarrow C$ is a colouring we say that $f$ is $\kappa$-bounded if and only if $\left|\left\{a \in[X]^{n}: f(a)=c\right\}\right| \leq \kappa$ for every $c \in C$, and $<\kappa$-bounded if and only if $\left|\left\{a \in[X]^{n}: f(a)=c\right\}\right|<\kappa$ for every $c \in C$.

Analogously to the usual notation for partition relations in classical Ramsey theory, we write $\alpha \rightarrow^{\text {poly }}(\beta)_{\kappa \text {-bdd }}^{n}$ for the assertion that every $\kappa$-bounded colouring of $[\alpha]^{n}$ has a polychromatic set of order type $\beta$. Galvin $[3,4]$ studied polychromatic Ramsey theory, and proved among other things the following results:

G1) For $m$ finite the classical relation $\alpha \rightarrow(\beta)_{m}^{n}$ implies the polychromatic relation $\alpha \rightarrow^{\text {poly }}(\beta)_{m-\text { bdd }}^{n}$.

G2) If $\kappa^{<\kappa}=\kappa$ then $\kappa \nrightarrow_{\text {poly }}(\kappa)_{2-\text { bdd }}^{2}$.

Using Galvin's result G1 and the classic result by Baumgartner and Hajnal [2] that $\omega_{1} \rightarrow(\alpha)_{2}^{2}$ for all $\alpha<\omega_{1}$, we get that $\omega_{1} \rightarrow^{\text {poly }}(\alpha)_{2-\text { bdd }}^{2}$ for all $\alpha<\omega_{1}$. Galvin's result G2 implies that under $\mathrm{CH} \omega_{1} \nrightarrow^{\text {poly }}\left(\omega_{1}\right)_{2-\text { bdd }}^{2}$. Todorčević [7] showed that the relation $\omega_{1} \rightarrow^{\text {poly }}\left(\omega_{1}\right)_{<\omega-\text { bdd }}^{2}$ is consistent relative to ZFC, and also that it follows from the Proper Forcing Axiom (PFA).

Cummings was partially supported by NSF grant DMS-0654046. Much of the research described in this paper was done while Cummings was visiting the Ben-Gurion University of the Negev in July 2008, as a Faculty of Natural Sciences Distinguished Scientist Visitors Program Visiting Fellow. He would like to acknowledge support from the visitors program, and to thank the Mathematics Department for its warm hospitality. 
In a previous paper [1] with Smyth, we studied polychromatic Ramsey theory for uncountable cardinals. The following are among the results of that paper:

ACS1) Martin's Axiom (MA) does not imply $\omega_{1} \rightarrow$ poly $\left(\omega_{1}\right)_{2-\text { bdd }}^{2}$.

ACS2) Martin's Maximum (MM) implies that every 2-bounded colouring of $\left[\omega_{2}\right]^{2}$ has a closed polychromatic set of order type $\omega_{1}$, but PFA does not suffice for this result.

ACS3) If $\kappa^{<\kappa}=\kappa$ then $\kappa^{+} \rightarrow^{\text {poly }}(\eta)_{<\kappa-\text { bdd }}^{2}$ for every $\eta<\kappa^{+}$.

Combining our result ACS3 with Galvin's result G2, we see that under GCH we get a fairly clear picture of which polychromatic relations hold (at least for successors of regular cardinals). Namely for $\kappa$ regular we get $\kappa^{+} \rightarrow^{\text {poly }}(\eta)_{<\kappa \text {-bdd }}^{2}$ for $\eta<\kappa^{+}$, and $\kappa^{+} \nrightarrow^{\text {poly }}\left(\kappa^{+}\right)_{2-\text { bdd }}^{2}$.

In this paper we continue to study polychromatic Ramsey theory without GCH, with our main focus being the cardinal $\omega_{2}$ with the hypothesis that $2^{\omega}=\omega_{2}$. It is easy to see that $\omega_{2} \rightarrow(\alpha)_{2-\text { bdd }}^{2}$ for every $\alpha<\omega_{1}$.

- In Section 2 we show (Theorem 1 ) that if $\kappa^{<\kappa}=\kappa$ the relation " $\kappa^{+} \rightarrow^{\text {poly }}$ $(\eta)_{<\kappa \text {-bdd }}^{2}$ for every $\eta<\kappa^{+"}$ holds in any $\kappa$-c.c. forcing extension. In particular (Corollary 1 ) if we add $\omega_{2}$ Cohen reals to a model of $\mathrm{CH}$ we get a model in which $\omega_{2} \rightarrow^{\text {poly }}(\alpha)_{\aleph_{0}-\text { bdd }}^{2}$ for every $\alpha<\omega_{2}$.

- In Section 3 we prove some technical facts about families of countable elementary substructures, for use in the forcing construction of Section 4.

- In Section 4 we show (Theorem 2) that assuming $\mathrm{CH}$ there is a proper $\aleph_{2^{-}}$ c.c. poset which adds a colouring witnessing the negative relation $\omega_{2} \nrightarrow^{\text {poly }}$ $\left(\omega_{1}\right)_{2-\text { bdd }}^{2}$.

- In Section 5 we describe some of the main open problems about polychromatic Ramsey theory for small uncountable cardinals.

\section{Models With LARGe PolyChromatic SETS}

As we mentioned in the introduction, in some previous joint work with Smyth [1] we proved that if $\kappa^{<\kappa}=\kappa$ then $\kappa^{+} \rightarrow^{\text {poly }}(\eta)_{<\kappa-\text { bdd }}^{2}$ for every $\eta<\kappa^{+}$. In this section we show, by a similar argument, that this polychromatic partition relation actually holds in every $\kappa$-c.c. forcing extension of a model in which $\kappa^{<\kappa}=\kappa$. This implies in particular that Theorem 2 of this paper could not be proved by c.c.c forcing.

We start by recalling some definitions and easy facts from [1]. If $f$ is a colouring of pairs from some ordered set $X$ then we say that $f$ is normal if $f\left(x_{1}, x_{2}\right)=f\left(y_{1}, y_{2}\right)$ implies $x_{2}=y_{2}$ whenever $x_{1}<x_{2}, y_{1}<y_{2}$. It is easy to see that if $f$ is any $<\kappa$-bounded colouring of pairs from $\kappa^{+}$, then there is an unbounded set $Z \subseteq \kappa^{+}$ such that $f \uparrow[Z]^{2}$ is normal. It follows that to prove $\kappa^{+} \rightarrow^{\text {poly }}(\eta)_{<\kappa-\text { bdd }}^{2}$ it will suffice to consider normal colourings.

Let $f$ be a $<\kappa$-bounded normal colouring of pairs from $\kappa^{+}$, and let $X \subseteq \kappa^{+}$. We define $F(X)$ to be the set of $\alpha<\kappa^{+}$such that there exist $\beta \in X$ and $\gamma \in X$ with $\alpha<\gamma, \beta<\gamma$ and $f(\alpha, \gamma)=f(\beta, \gamma)$. The proof of the following lemma is routine:

Lemma 1. Let $f$ and $F$ be as above.

(1) If $|X|<\kappa$ then $|F(X)|<\kappa$.

(2) Let $X<\alpha<Y$ where $X \cup\{\alpha\}$ is polychromatic for $f, X \cup Y$ is polychromatic for $f$, and $\alpha \notin F(X \cup Y)$. Then $X \cup\{\alpha\} \cup Y$ is polychromatic for $f$. 
In a mild abuse of terminology we will refer to $F$ as the Skolem function of $f$.

Theorem 1. Let $\kappa^{<\kappa}=\kappa$ and let $\mathbb{P}$ be any $\kappa$-c.c. forcing poset. Then for every $\eta<\kappa^{+}$, the polychromatic partition relation $\kappa^{+} \rightarrow^{\text {poly }}(\eta)_{<\kappa-\text { bdd }}^{2}$ holds in the generic extension by $\mathbb{P}$.

Proof. Fix $\eta<\kappa^{+}$. Without loss of generality we may assume that $\eta$ is a limit ordinal and $\eta \geq \kappa$. We fix a bijection $g: \kappa \simeq \eta$. Let $\dot{f}$ be a $\mathbb{P}$-name for a $<\kappa$ bounded normal colouring of pairs from $\kappa^{+}$, and let $\dot{F}$ be a $\mathbb{P}$-name for the Skolem function of $\dot{f}$. Since $\mathbb{P}$ is $\kappa$-c.c, there is in $V$ a function $F^{*}:\left[\kappa^{+}\right]^{<\kappa} \rightarrow\left[\kappa^{+}\right]^{<\kappa}$ such that $\Vdash_{\mathbb{P}} \dot{F}(A) \subseteq F^{*}(A)$ for all $A \in\left[\kappa^{+}\right]<\kappa$.

The idea of the proof is that we will build in $V$ a set $A$ of order type $\eta$, which is forced by $\mathbb{P}$ to be polychromatic for the colouring named by $\dot{f}$. The set $A$ will be built in an inductive construction of length $\kappa$, where at stage $i$ we will determine the $g(i)^{\text {th }}$ ordinal in the increasing enumeration of $A$. To this end, we fix a large regular cardinal $\theta$ and a sequence $\left\langle M_{i}: i \leq \eta\right\rangle$ such that:

(1) $M_{i} \prec H_{\theta}$ and $\left|M_{i}\right|=\kappa$ for all $i \leq \eta$.

(2) $M_{i} \cup\left\{M_{i}\right\} \subseteq M_{i+1}$ for all $i<\eta$.

(3) $\left\{\mathbb{P}, \dot{f}, \dot{F}, F^{*}\right\} \cup \kappa \subseteq M_{0}$.

(4) ${ }^{<\kappa} M_{i} \subseteq M_{i}$ for all successor $i \leq \eta$.

(5) $M_{j}=\bigcup_{i<j} M_{i}$ for all limit $j \leq \eta$.

Let $\lambda_{i}=M_{i} \cap \kappa^{+}$. It is easy to see that $\lambda_{i}<\kappa^{+}, \lambda_{i}$ is strictly increasing with $i$, and $c f\left(\lambda_{i}\right)=\kappa$ for every successor $i$. Let $\alpha$ be such that $\lambda_{\eta}<\alpha<\kappa^{+}$. We will choose by induction ordinals $\left\langle\alpha_{i}: i<\kappa\right\rangle$ maintaining the hypotheses that for every $i<\kappa$ :

(1) $\lambda_{g(i)}<\alpha_{i}<\lambda_{g(i)+1}$

(2) $\left\{\alpha_{j}: j<i\right\} \cup\{\alpha\}$ is forced by $\mathbb{P}$ to be polychromatic for $\dot{f}$.

Suppose that we have chosen $\alpha_{j}$ for $j<i$. Let $X=\left\{\alpha_{j}: j<i, g(j)<g(i)\right\}$ and $Y=\left\{\alpha_{j}: j<i, g(j)>g(i)\right\} \cup\{\alpha\}$. Since ${ }^{<\kappa} M_{g(i)+1} \subseteq M_{g(i)+1}$, we see that $X \in M_{g(i)+1}$. Now note that by item 2 above, $\alpha$ has the property " $X \cup\{\alpha\}$ is forced by $\mathbb{P}$ to be polychromatic for $\dot{f}$ ". Since $M_{g(i)+1} \prec H_{\theta}$ and the parameters $X, \mathbb{P}, \dot{f}$ are all in $M_{g(i)+1}$, while $\alpha>\lambda_{g(i)+1}=M_{g(i)+1} \cap \kappa^{+}$, a standard application of elementarity gives us that there are unboundedly many $\beta<\lambda_{g(i)+1}$ such that $X \cup\{\beta\}$ is forced by $\mathbb{P}$ to be polychromatic for $\dot{f}$.

Now $X \cup Y=\left\{\alpha_{j}: j<i\right\}$, a set of size less than $\kappa$, so if we let $Z=F^{*}(X \cup Y)$ then $|Z|<\kappa$ and $\Vdash \dot{F}(X \cup Y) \subseteq Z$. We now choose $\alpha_{i}$ to be some $\beta<\lambda_{g(i)+1}$ such that $X<\beta, \lambda_{g(i)}<\beta, \beta \notin Z$, and $X \cup\{\beta\}$ is forced by $\mathbb{P}$ to be polychromatic for $\dot{f}$. This is possible, since the last of these properties holds for unboundedly many $\beta$.

By construction it is forced by $\mathbb{P}$ that $X \cup Y$ is polychromatic for $\dot{f}, X \cup\left\{\alpha_{i}\right\}$ is polychromatic for $\dot{f}$, and $\alpha_{i} \notin \dot{F}(X \cup Y)$. Appealing to Lemma 1 in the generic extension by $\mathbb{P}$, it is forced that $X \cup\left\{\alpha_{i}\right\} \cup Y$ (that is $\left\{\alpha_{j}: j \leq i\right\} \cup\{\alpha\}$ ) is polychromatic for $\dot{f}$, so that we have carried the induction forward by one step.

When the induction is finished we let $A=\left\{\alpha_{i}: i<\kappa\right\}$. The construction guaranteed that $\lambda_{g(i)}<\alpha_{i}<\lambda_{g(i)+1}$, so that $o t(A)=\eta$. Also it is forced by $\mathbb{P}$ that $A$ is polychromatic for $\dot{f}$. 
Corollary 1. In any c.c.c. extension of a model of CH, $\omega_{2} \rightarrow^{\text {poly }}(\eta)_{\aleph_{0}-b d d}^{2}$ for every $\eta<\omega_{2}$.

By contrast the conclusion of Theorem 2 is that the negative relation $\omega_{2} \nrightarrow_{\nrightarrow}$ poly $\left(\omega_{1}\right)_{2-b d d}^{2}$ is consistent, and it follows from Corollary 1 that this result can not be obtained by c.c.c. forcing.

\section{NiCELY ARRANGED FAMILIES OF MODELS}

We will use the forcing technique of models as side conditions, which was introduced by Todorčević [8]. This is a general technique for constructing proper forcing posets. In its most basic form the idea is as follows: a forcing condition will be a pair $(p, \vec{M})$ where $p$ is the "working part" and $\vec{M}$ is a finite $\epsilon$-chain of countable elementary submodels of $H_{\kappa}$ for some fixed $\kappa$. Properness is typically proved in the following way; if $(p, \vec{M}) \in N$ where $N$ is a countable submodel of $H_{\lambda}$ for some $\lambda>\kappa$, then $\left(p, \vec{M} \frown\left(N \cap H_{\kappa}\right)\right)$ is a an $N$-generic condition extending $(p, \vec{M})$. For example, the proof of Theorem 2 in our previous paper [1] on polychromatic Ramsey theory uses a forcing construction of exactly this kind.

Forcing posets constructed with chains of models as side conditions typically do not have the $\aleph_{2}$-c.c. The main issue here is that when we are given a list of conditions $\left(p_{i}, \vec{M}_{i}\right)$ for $i<\omega_{2}$, the best we can do (even assuming $\mathrm{CH}$ ) is to find $i<j$ where the chains $\vec{M}_{i}, \vec{M}_{j}$ are of the same length and consist of pairwise isomorphic but unequal models; this is an insuperable obstacle to amalgamating $\left(p_{i}, \vec{M}_{i}\right)$ and $\left(p_{j}, \vec{M}_{j}\right)$. The remedy, which again is due to Todorčević [9], is to use side conditions which are finite matrices of models. This idea has been used by several authors $[6,5]$, and will be a key point in the proof of Theorem 2 .

In this section we define nicely arranged families of models, and prove their basic properties. These families will be the side conditions used in the forcing poset for Theorem 2. Many of the results from this section already appear (at least implicitly) in the literature on forcing with matrices of models as side conditions, but still they seem worth isolating in an explicit form divorced from the discussion of any particular forcing poset.

We fix a well-ordering $<$ of the set $H_{\omega_{2}}$. In a mild abuse of notation we will talk about "elementary substructures of $H_{\omega_{2}}$ " when we really mean "elementary substructures of the structure $\left(H_{\omega_{2}}, \in,<\right)$ ". For each $a \in H_{\omega_{2}}$, let $f_{a} \in H_{\omega_{2}}$ be the $<$-least bijection between $a$ and $|a|$, where we note that $|a| \leq \omega_{1}$.

We denote the set of countable elementary substructures of $H_{\omega_{2}}$ by $\mathcal{C}$. If $K \in \mathcal{C}$, then by the Mostowski collapsing lemma $K$ is isomorphic to a unique transitive set $\bar{K}$, via a unique isomorphism which we denote by $\pi_{K}: K \simeq \bar{K}$. For $K \in \mathcal{C}$ we denote the countable ordinal $K \cap \omega_{1}$ by $\delta_{K}$, where we note that $\omega_{1} \in K$ and $\delta_{K}=\pi_{K}\left(\omega_{1}\right)=\omega_{1}{ }^{\bar{K}}$.

Notation: In the sequel we generally reserve upper case roman letters in the middle of the alphabet for countable elementary substructures of some $H_{\kappa}$, and lower case roman letters towards the end of the alphabet for their transitive collapses.

Lemma 2. Let $K, L \in \mathcal{C}$. Then

(1) If $L \in K$ then $L \subseteq K$ and $\delta_{L}<\delta_{K}$.

(2) If $K$ and $L$ are isomorphic then $\delta_{K}=\delta_{L}$, there is a unique isomorphism between $K$ and $L$, and this isomorphism fixes every element of $K \cap L$. 
Proof. Since $\omega \subseteq K$, every countable element of $K$ is a subset of $K$, and the first claim follows immediately. If $K$ and $L$ are isomorphic, then $\bar{K}=\bar{L}$, and a routine induction on rank shows that if $\pi=\pi_{L}^{-1} \circ \pi_{K}$ then $\pi$ is the unique isomorphism between $K$ and $L$. As we noted above $\pi_{K}\left(\omega_{1}\right)=\delta_{K}=\omega_{1}^{\bar{K}}$, and similarly $\delta_{L}=\omega_{1}^{\bar{L}}$, and so $\delta_{K}=\delta_{L}$ since $\bar{K}=\bar{L}$.

We claim that for every $a \in K \cap L, a \cap K=a \cap L$. To see this observe that $f_{a} \in K \cap L$ and $|a| \leq \omega_{1}$, so $f_{a} "(a \cap K)=|a| \cap K=|a| \cap L=f_{a} "(a \cap L)$, and hence $a \cap K=a \cap L$. An easy induction on rank now shows that $\pi_{K}\left\lceil K \cap L=\pi_{L}\lceil K \cap L\right.$, and since $\pi=\pi_{L}^{-1} \circ \pi_{K}$ we have $\pi \uparrow K \cap L=i d$.

When $K$ and $L$ are isomorphic, we denote the unique isomorphism between them by $\pi_{K L}: K \simeq L$.

Lemma 3. Let $K, L, M \in \mathcal{C}$, and assume that $K \simeq L$ and $M \in K$. Let $N=$ $\pi_{K L}(M)$. Then $N=\pi_{K L} " M, N \in \mathcal{C}, N \simeq M$ and $\pi_{M N}=\pi_{K L} \uparrow M$.

Proof. Since $M \in K$ and $M$ is countable there is a bijection $f: \omega \rightarrow M$ with $f \in K$. Then $\pi_{K L}(f)$ is a bijection between $\omega$ and $N$, and $\pi_{K L}(f(n))=\pi_{K L}(f)(n)$ for all $n$, so $N=\pi_{K L}$ " $M$. Since $M \subseteq K$ and $M, K \prec H_{\omega_{2}}$ we have that $M \prec K$, and so $N \prec L$ since $N=\pi_{K L}$ " $M$. Since $L \prec H_{\omega_{2}}$ we have $N \prec H_{\omega_{2}}$, so that $N \in \mathcal{C}$. Finally $\pi_{K L} \uparrow M$ is an isomorphism between $M$ and $N$, and so $\pi_{M N}=\pi_{K L} \uparrow M$ by the uniqueness of isomorphisms.

The following definition abstracts certain properties of the families of models used as side conditions in papers by Todorčević [9], Shelah and Zapletal [6] and Koszmider [5]. We have in mind particularly the side conditions for the forcing $\mathcal{P}_{D}$ in [9, section 4], the matrices of models in [6, Definition 18], and the side conditions for the forcing $P$ in [5, Section 3].

Definition 1. A nicely arranged family is a finite set $\mathcal{F} \subseteq \mathcal{C}$ such that

(1) For all $M, N \in \mathcal{F}$, if $\delta_{M}=\delta_{N}$ then $M \simeq N$.

(2) For all $M, N \in \mathcal{F}$, if $\delta_{M}<\delta_{N}$ then there is $N^{\prime} \in \mathcal{F}$ such that $N^{\prime} \simeq N$ and $M \in N$.

(3) For all $M, N \in \mathcal{F}$ with $M \simeq N, \pi_{M N}$ " $(M \cap \mathcal{F})=N \cap \mathcal{F}$.

Let $\mathcal{F}$ be nicely arranged. If $M, N \in \mathcal{F}$ with $\delta_{M}=\delta_{N}$ then it follows easily from the definition that $\bar{M}=\bar{N}$, and also that $\pi_{M} "(M \cap \mathcal{F})=\pi_{N} "(N \cap \mathcal{F})$.

Definition 2. Let $\mathcal{F}$ be nicely arranged, and let $\left\langle\delta_{i}: i\langle n\rangle\right.$ be the enumeration of $\left\{\delta_{M}: M \in \mathcal{F}\right\}$ in increasing order.

(1) The height of $\mathcal{F}$ is the natural number $n$.

(2) The type of $\mathcal{F}$ is the finite sequence $\left\langle\left(x_{i}, m_{i}\right): i\langle n\rangle\right.$ such that $\bar{M}=x_{i}$ and $\pi_{M}$ " $(M \cap \mathcal{F})=m_{i}$ for all $M \in \mathcal{F}$ with $\delta_{M}=\delta_{i}$.

Lemma 4. Let $\mathcal{F}$ be nicely arranged. Let $K, L \in \mathcal{F}$ with $\delta_{K}<\delta_{L}$. Then there is $K^{\prime} \in \mathcal{F}$ with $K^{\prime} \in L$ and $K^{\prime} \simeq K$.

Proof. By clause 2 of Definition 1 , there is $L^{\prime} \in \mathcal{F}$ such that $K \in L^{\prime}$ and $L^{\prime} \simeq L$. Let $K^{\prime}=\pi_{L^{\prime} L}(K)$. By Lemma $3 K^{\prime} \simeq K$, and by clause 3 of Definition $1 K^{\prime} \in \mathcal{F}$.

We note that since nicely arranged families are finite, if $\mathcal{F}$ is nicely arranged and $M \in \mathcal{C}$ then $\mathcal{F} \in M$ if and only if $\mathcal{F} \subseteq M$. It is also clear that if $\mathcal{F} \in M$ and $M^{\prime} \in \mathcal{C}$ with $M \simeq M^{\prime}$, then $\pi_{M M^{\prime}}(\mathcal{F})$ is nicely arranged. 
Lemma 5. Let $\mathcal{F}$ be nicely arranged and let $M \in \mathcal{F}$. Then $M \cap \mathcal{F}$ is nicely arranged.

Proof. Clause 1 of Definition 1 is immediate. To verify clause 2, let $K, L \in M \cap \mathcal{F}$ with $\delta_{K}<\delta_{L}$. By clause 2 for $\mathcal{F}$, there is $L^{\prime} \in \mathcal{F}$ with $L^{\prime} \simeq L$ and $K \in L^{\prime}$. Now $\delta_{L^{\prime}}=\delta_{L}<\delta_{M}$, so applying clause 2 for $\mathcal{F}$ again there is $M^{\prime} \in \mathcal{F}$ with $M^{\prime} \simeq M$ and $L^{\prime} \in M^{\prime}$. Let $L^{\prime \prime}=\pi_{M^{\prime} M}\left(L^{\prime}\right)$, and note that $L^{\prime \prime} \in M, L^{\prime \prime} \simeq L^{\prime}$ and that $L^{\prime \prime} \in \mathcal{F}$ by clause 3 for $\mathcal{F}$. Now $K \in L^{\prime}$ and $L^{\prime} \subseteq M^{\prime}$, so $K \in M^{\prime}$; also $K \in M$ by hypothesis, and so $\pi_{M^{\prime} M}(K)=K$ by clause 2 of Lemma 2 . Since $K \in L^{\prime}$, it follows by applying the isomorphism $\pi_{M^{\prime} M}$ that $K \in L^{\prime \prime}$.

Finally let $K, L \in M \cap \mathcal{F}$ with $K \simeq L$. By clause 3 of Definition $1, \pi_{K L}$ " $(K \cap \mathcal{F})=$ $L \cap \mathcal{F}$. Since $K \in M$ we have $K \subseteq M$, so that $K \cap(M \cap \mathcal{F})=K \cap \mathcal{F}$, and similarly $L \cap(M \cap \mathcal{F})=L \cap \mathcal{F}$. It follows immediately that clause 3 is true for $M \cap \mathcal{F}$.

We are now almost ready to prove two technical lemmas, which are the main goal of this section. The Diffusion Lemma (Lemma 7) is useful for proving properness, while the Amalgamation Lemma (Lemma 8) is useful for proving the $\aleph_{2}$-c.c.

Definition 3. Let $\mathcal{F}$ be nicely arranged, and let $M \in \mathcal{F}$. Let $\mathcal{G}$ be a nicely arranged set such that $M \cap \mathcal{F} \subseteq \mathcal{G} \subseteq M$. Then the diffusion of $\mathcal{G}$ from $M$ in $\mathcal{F}$ is the set

$\operatorname{Diff}(\mathcal{G}, M, \mathcal{F})=_{\operatorname{def}}\left\{N^{\prime} \in \mathcal{F}: \delta_{N^{\prime}} \geq \delta_{M}\right\} \cup\left\{\pi_{M M^{\prime}}(N): N \in \mathcal{G}, M^{\prime} \in \mathcal{F}, M \simeq M^{\prime}\right\}$.

We note that if $N^{\prime} \in \operatorname{Diff}(\mathcal{G}, M, \mathcal{F})$ and $\delta_{N^{\prime}} \geq \delta_{M}$ then $N^{\prime} \in \mathcal{F}$, while if $\delta_{N^{\prime}}<\delta_{M}$ then $N^{\prime}=\pi_{M M^{\prime}}(N)$ for some $M^{\prime} \in \mathcal{F}$ and some $N \in \mathcal{G}$. We will soon prove that $\operatorname{Diff}(\mathcal{G}, M, \mathcal{F})$ is the least nicely arranged set containing $\mathcal{F} \cup \mathcal{G}$; we begin by arguing that $\operatorname{Diff}(\mathcal{G}, M, \mathcal{F})$ contains $\mathcal{F} \cup \mathcal{G}$, and that the "base model" $M$ can be replaced by any $M^{\prime} \in \mathcal{F}$ which is isomorphic to $M$.

Lemma 6. Let $\mathcal{F}$ and $\mathcal{G}$ be nicely arranged sets with $M \cap \mathcal{F} \subseteq \mathcal{G} \subseteq M$ for some $M \in \mathcal{F}$. Let $\mathcal{H}=\operatorname{Diff}(\mathcal{G}, M, \mathcal{F})$. Then

(1) $\mathcal{H}$ contains $\mathcal{F} \cup \mathcal{G}$.

(2) Let $M^{\prime} \in \mathcal{F}$ with $M^{\prime} \simeq M$. Then

(a) $M^{\prime} \cap \mathcal{H}=\pi_{M M^{\prime}}$ ' $\mathcal{G}$, and in particular $M \cap \mathcal{H}=\mathcal{G}$.

(b) If $\mathcal{G}^{\prime}=M^{\prime} \cap \mathcal{H}$ then $\mathcal{G}^{\prime}$ is nicely arranged, $M^{\prime} \cap \mathcal{F} \subseteq \mathcal{G}^{\prime} \subseteq M^{\prime}$, and $\mathcal{H}=\operatorname{Diff}\left(\mathcal{G}^{\prime}, M^{\prime}, \mathcal{F}\right)$.

Proof. We take the claims in turn.

(1) By definition $\mathcal{H}$ contains all $N^{\prime} \in \mathcal{F}$ with $\delta_{N^{\prime}} \geq \delta_{M}$. If $N^{\prime} \in \mathcal{F}$ and $\delta_{N^{\prime}}<\delta_{M}$, then $N^{\prime} \in M^{\prime}$ for some $M^{\prime} \in \mathcal{F}$ with $M^{\prime} \simeq M$. Then $\pi_{M^{\prime} M}\left(N^{\prime}\right) \in M \cap \mathcal{F}$, and so by hypothesis $\pi_{M^{\prime} M}\left(N^{\prime}\right) \in \mathcal{G}$, and then since $N^{\prime}=\pi_{M M^{\prime}}\left(\pi_{M^{\prime} M}\left(N^{\prime}\right)\right)$ we have $N^{\prime} \in \mathcal{H}$. Finally if $N \in \mathcal{G}$ then $N=\pi_{M M}(N)$ so that $N \in \mathcal{H}$.

(2) It is clear from the definition of diffusion that $\pi_{M M^{\prime}}$ " $\mathcal{G} \subseteq M^{\prime} \cap \mathcal{H}$. For the reverse inclusion, let $N^{\prime} \in M^{\prime} \cap \mathcal{H}$. Since $\delta_{N^{\prime}}<\delta_{M^{\prime}}=\delta_{M}, N^{\prime}=\pi_{M M^{\prime \prime}}(N)$ for some $N \in \mathcal{G}$ and $M^{\prime \prime} \in \mathcal{F}$. Now $N^{\prime} \in M^{\prime} \cap M^{\prime \prime}$, so $N^{\prime}$ is fixed by $\pi_{M^{\prime \prime} M^{\prime}}$, thus $N^{\prime}=\pi_{M M^{\prime}}(N)$.

Lemma 7 (Diffusion lemma). Let $\mathcal{F}$ and $\mathcal{G}$ be nicely arranged sets with $M \cap \mathcal{F} \subseteq$ $\mathcal{G} \subseteq M$ for some $M \in \mathcal{F}$. Let $\mathcal{H}=\operatorname{Diff}(\mathcal{G}, M, \mathcal{F})$. Then

(1) $\mathcal{H}$ is nicely arranged. 
(2) $\mathcal{H}$ is the least nicely arranged set which contains $\mathcal{F} \cup \mathcal{G}$.

Proof. We have already shown that $\mathcal{H} \supseteq \mathcal{F} \cup \mathcal{G}$, and it is clear from the definition of "nicely arranged" that any nicely arranged family containing $\mathcal{F} \cup \mathcal{G}$, contains $\mathcal{H}$. So it will suffice to verify that $\mathcal{H}$ is nicely arranged. To show that $\mathcal{H}$ is nicely arranged, we verify each clause in Definition 1.

Clause 1: Suppose that $N_{1}, N_{2} \in \mathcal{H}$ with $\delta_{N_{1}}=\delta_{N_{2}}$; we must show that $N_{1} \simeq N_{2}$. If $\delta_{N_{1}}=\delta_{N_{2}} \geq \delta_{M}$, then $N_{1}, N_{2} \in \mathcal{F}$, so that $N_{1} \simeq N_{2}$ by clause 1 for $\mathcal{F}$. So we assume that $\delta_{N_{1}}=\delta_{N_{2}}<\delta_{M}$; by part 2 of Lemma 6 we may assume without loss of generality that $N_{1} \in \mathcal{G}$, while $N_{2}=\pi_{M M^{\prime}}\left(N_{2}^{\prime}\right)$ for some $M^{\prime} \in \mathcal{F}$ and $N_{2}^{\prime} \in \mathcal{G}$. Now $\delta_{N_{1}}=\delta_{N_{2}}=\delta_{N_{2}^{\prime}}$, and clause 1 for $\mathcal{G}$ implies that $N_{1} \simeq N_{2}^{\prime}$; since also $N_{2} \simeq N_{2}^{\prime}$, we conclude that $N_{1} \simeq N_{2}$.

Clause 2: Suppose that $K, L \in \mathcal{H}$ with $\delta_{K}<\delta_{L}$; we must show that $K \in L^{\prime}$ for some $L^{\prime} \in \mathcal{H}$ with $L^{\prime} \simeq L$. We distinguish various cases:

Case 1: $\delta_{M} \leq \delta_{K}<\delta_{L}$. In this case both $K, L$ are in $\mathcal{F}$, so the claim follows immediately from clause 2 for $\mathcal{F}$.

Case 2: $\delta_{K}<\delta_{M} \leq \delta_{L}$. By part 2 of Lemma 6 , we may assume without loss of generality that $K \in \mathcal{G}$, in particular $K \in M$. If $\delta_{M}=\delta_{L}$ then we may set $L^{\prime}=M$. If $\delta_{M}<\delta_{L}$ then $M \in L^{\prime}$ for some $L^{\prime} \in \mathcal{F}$ such that $L^{\prime} \simeq L$, and we are done since $K \in M \subseteq L^{\prime}$.

Case 3: $\delta_{K}<\delta_{L}<\delta_{M}$. We may assume that $K \in \mathcal{G}$, and that $L=\pi_{M M^{\prime}}\left(L^{\prime}\right)$ for some $L^{\prime} \in \mathcal{G}$. By clause 2 for $\mathcal{G}$, there is $L^{\prime \prime} \in \mathcal{G}$ such that $K \in L^{\prime \prime}$ and $L^{\prime \prime} \simeq L^{\prime}$. We are done since $L^{\prime} \simeq L$ and $L^{\prime \prime} \in \mathcal{H}$.

Clause 3: Suppose that $K, L \in \mathcal{H}$ with $K \simeq L$ and $J \in K \cap \mathcal{H}$. We must show that $\pi_{K L}(J) \in \mathcal{H}$.

Case 1: $\delta_{M} \leq \delta_{J}<\delta_{K}=\delta_{L}$. The conclusion is immediate since $J, K, L \in \mathcal{F}$ and $\pi_{K L} "(K \cap \mathcal{F})=(L \cap \mathcal{F})$.

Case 2: $\delta_{J}<\delta_{M}$. Without loss of generality $J \in \mathcal{G}$, in particular $J \in M$.

Subcase 2a: $\delta_{K}=\delta_{L}=\delta_{M}$. Since $J \in K \cap M, J$ is fixed by $\pi_{M K}$. So $\pi_{K L}(J)=\pi_{M L}(J)$, and hence $\pi_{K L}(J) \in \mathcal{H}$.

Subcase 2b: $\delta_{K}=\delta_{L}>\delta_{M}$. Find $K^{\prime} \in \mathcal{F}$ such that $M \in K^{\prime}$. Since $M \subseteq K^{\prime}, J \in K^{\prime}$. Since $J \in K^{\prime} \cap K$ it is fixed by $\pi_{K^{\prime} K}$, so $\pi_{K L}(J)=\pi_{K^{\prime} L}(J)$. Now let $M^{\prime}=\pi_{K^{\prime} L}(M)$, so that $M^{\prime} \simeq M$ and $\pi_{K^{\prime} L} \uparrow M=\pi_{M M^{\prime}}$; in particular $\pi_{K L}(J)=\pi_{K^{\prime} L}(J)=\pi_{M M^{\prime}}(J)$ and we are done by subcase 2 a.

Subcase 2c: $\delta_{K}=\delta_{L}<\delta_{M}$. We have $K=\pi_{M M^{\prime}}\left(K^{\prime}\right)$ and $L=\pi_{M M^{\prime \prime}}\left(L^{\prime}\right)$ for some $M^{\prime}, M^{\prime \prime} \in \mathcal{F}$ and $K^{\prime}, L^{\prime} \in \mathcal{G}$. Note that $\pi_{K K^{\prime}}=\pi_{M^{\prime} M} \uparrow K$, and similarly $\pi_{L^{\prime} L}=\pi_{M M^{\prime \prime}} \uparrow L^{\prime}$. We factorise $\pi_{K L}$ as $\pi_{L^{\prime} L} \circ \pi_{K^{\prime} L^{\prime}} \circ \pi_{K K^{\prime}}$ Since $J \in M$ and $J \in K \subseteq M^{\prime}$, we have $J \in M \cap M^{\prime}$ and $\pi_{K K^{\prime}}(J)=\pi_{M^{\prime} M}(J)=J$. By clause 3 for the nicely arranged family $\mathcal{G}, \pi_{K L^{\prime}}(J)=\pi_{K^{\prime} L^{\prime}}(J) \in \mathcal{G}$. Finally by Subcase 2a $\pi_{K L}(J)=\pi_{L L^{\prime}}\left(\pi_{K^{\prime} L}(J)\right)=\pi_{M M^{\prime \prime}}\left(\pi_{K^{\prime} L}(J)\right) \in \mathcal{H}$, and we are done.

Lemma 8 (Amalgamation lemma). Let $\mathcal{F}_{0}$ and $\mathcal{F}_{1}$ be two nicely arranged systems with the same type, and let $\mathcal{F}=\mathcal{F}_{0} \cup \mathcal{F}_{1}$. Then $\mathcal{F}$ is nicely arranged and has the same type as $\mathcal{F}_{0}$ and $\mathcal{F}_{1}$.

Proof. The proofs of clauses 1 and 2 from Definition 1 are very straightforward, so we only prove clause 3 . We claim first that for $i \in\{0,1\}$ and $L \in \mathcal{F}_{i}, L \cap \mathcal{F}=L \cap \mathcal{F}_{i}$. By symmetry it will suffice to show that $L \cap \mathcal{F}_{0} \subseteq \mathcal{F}_{1}$ for all $L \in \mathcal{F}_{1}$. 
Suppose that $K \in \mathcal{F}_{0}$ and $L \in \mathcal{F}_{1}$, with $K \in L$. Since the types agree there is $L^{\prime} \in \mathcal{F}_{0}$ with $L^{\prime} \simeq L$, and since $\mathcal{F}_{0}$ is nicely arranged we may assume that $K \in L^{\prime}$. What is more the agreement of types implies that $\pi_{L^{\prime}}$ " $\left(L^{\prime} \cap \mathcal{F}_{0}\right)=\pi_{L}$ " $L \cap \mathcal{F}_{1}$, In particular since $K \in L^{\prime} \cap \mathcal{F}_{0}$, we have $\pi_{L^{\prime}}(K)=\pi_{L}\left(K^{\prime}\right)$ for some $K^{\prime} \in L \cap \mathcal{F}_{1}$, so that $K^{\prime}=\pi_{L L^{\prime}}(K)$. Now $K \in L \cap L^{\prime}$, so it is fixed by $\pi_{L L^{\prime}}$ and thus $K=K^{\prime} \in \mathcal{F}_{1}$. So $L \cap \mathcal{F}_{0} \subseteq \mathcal{F}_{1}$ as claimed.

Now suppose that $K, L$ are isomorphic structures in $\mathcal{F}$. It follows from the claim above and the agreement of types that $\pi_{K} "(K \cap \mathcal{F})=\pi_{L} "(L \cap \mathcal{F})$, so that $\pi_{K L} "(K \cap \mathcal{F})=L \cap \mathcal{F}$. It is immediate from this argument that $\mathcal{F}$ has the same type as $\mathcal{F}_{0}$ and $\mathcal{F}_{1}$.

\section{Models without large Polychromatic SETS}

In this section we will prove our main result, the consistency of the negative polychromatic relation $\omega_{2} \nrightarrow$ poly $\left(\omega_{1}\right)_{2-b d d}^{2}$. Our model will be obtained as a proper $\aleph_{2}$-c.c. extension of a model of $\mathrm{CH}$. Accordingly we assume for the rest of this section that $\mathrm{CH}$ holds in $V$.

The generic object will actually be a function $f:\left[\omega_{2}\right]^{2} \rightarrow \omega_{1}$, with properties that will enable us to derive a colouring $g$ which witnesses $\omega_{2} \nrightarrow^{\text {poly }}\left(\omega_{1}\right)_{2-b d d}^{2}$ by setting $g(\alpha, \beta)=(f(\alpha, \beta), \beta)$. We note that this is rather natural in the light of the discussion of normal functions at the start of Section 2, which implies in particular that if we can find a function $g$ to witness $\omega_{2} \nrightarrow^{\text {poly }}\left(\omega_{1}\right)_{2-b d d}^{2}$ then we can find one derived from a suitable $f$ in this way. Similar ideas were used in our previous paper [1] to create generic "bad" colourings of pairs from $\omega_{1}$.

To be more precise we will add $f$ such that

F1) $f:\left[\omega_{2}\right]^{2} \rightarrow \omega_{1}$.

F2) There do not exist ordinals $\alpha_{0}, \alpha_{1}, \alpha_{2}, \beta$ such that $\alpha_{0}<\alpha_{1}<\alpha_{2}<\beta$ and $f\left(\alpha_{0}, \beta\right)=f\left(\alpha_{1}, \beta\right)=f\left(\alpha_{2}, \beta\right)$.

F3) For any $X \subseteq \omega_{2}$ of order type $\omega_{1}$, there exist $\alpha_{0}, \alpha_{1}, \beta \in X$ such that $\alpha_{0}<\alpha_{1}<\beta$ and $f\left(\alpha_{0}, \beta\right)=f\left(\alpha_{1}, \beta\right)$.

It is routine to check that if $f$ has these properties, and we let $g(\alpha, \beta)=(f(\alpha, \beta), \beta)$, then $g$ is a 2-bounded colouring with no polychromatic set of order type $\omega_{1}$.

We are now ready to describe our forcing poset $\mathbb{P}$. Recall from Section 3 that we fixed a well-ordering $<$ of $H_{\omega_{2}}$, and that $f_{\alpha}$ is the <-least bijection between $\alpha$ and $|\alpha|$ for every $\alpha<\omega_{2}$. In particular $f_{\alpha}$ is an injective map from $\alpha$ to $\omega_{1}$.

(1) The conditions in $\mathbb{P}$ are pairs $p=\left(c_{p}, \mathcal{F}_{p}\right)$ where

(a) $c_{p}$ is a finite partial function from $\left[\omega_{2}\right]^{2}$ to $\omega_{1}$.

(b) For every $(\alpha, \beta) \in \operatorname{dom}\left(c_{p}\right), c_{p}(\alpha, \beta) \geq f_{\beta}(\alpha)$.

(c) There do not exist ordinals $\alpha_{0}, \alpha_{1}, \alpha_{2}, \beta$ such that $\alpha_{0}<\alpha_{1}<\alpha_{2}<$ $\beta,\left\{\left(\alpha_{0}, \beta\right),\left(\alpha_{1}, \beta\right),\left(\alpha_{2}, \beta\right)\right\} \subseteq \operatorname{dom}\left(c_{p}\right)$, and $c_{p}\left(\alpha_{0}, \beta\right)=c_{p}\left(\alpha_{1}, \beta\right)=$ $c_{p}\left(\alpha_{2}, \beta\right)$.

(d) $\mathcal{F}_{p}$ is a nicely arranged family.

(2) Let $p=\left(c_{p}, \mathcal{F}_{p}\right)$ and $q=\left(c_{q}, \mathcal{F}_{q}\right)$ be elements of $\mathbb{P}$. Then $q \leq p$ ( $q$ is stronger than $p$ ) if and only if

(a) $\operatorname{dom}\left(c_{p}\right) \subseteq \operatorname{dom}\left(c_{q}\right)$, and $c_{q}\left\lceil\operatorname{dom}\left(c_{p}\right)=c_{p}\right.$.

(b) $\mathcal{F}_{p} \subseteq \mathcal{F}_{q}$.

(c) For every $(\alpha, \beta) \in \operatorname{dom}\left(c_{q}\right) \backslash \operatorname{dom}\left(c_{p}\right)$ and every $M \in \mathcal{F}_{p}$, if $(\alpha, \beta) \in M$ then $c_{q}(\alpha, \beta) \in M$. 
Before we start the detailed analysis of $\mathbb{P}$ we make some remarks about the definition of this forcing poset.

(1) The "working part" $c$ of the condition $(c, \mathcal{F})$ is a finite approximation to the generic function $f$. The models in $\mathcal{F}$ are auxiliary objects whose job is to enforce properness. Clause 2c) in the definition of extension and the demand that $c_{p}(\alpha, \beta) \geq f_{\beta}(\alpha)$ will be used in the proof of properness, see in particular Claims 3 and 4 in the proof of Lemma 12.

(2) For a condition $(c, \mathcal{F})$ we do not demand that the structures in $\mathcal{F}$ should be closed under the partial function $c$, nor that the isomorphisms between structures in $\mathcal{F}$ should respect $c$ in any way.

(3) When $p$ and $q$ are conditions and $q$ extends $p$, our only demand involves structures in $\mathcal{F}_{p}$ and pairs in $\operatorname{dom}\left(c_{q}\right) \backslash \operatorname{dom}\left(c_{p}\right)$. In particular the structures in $\mathcal{F}_{q} \backslash \mathcal{F}_{p}$ are not required to be closed even under $c_{p}$.

Lemma 9. Let $p \in \mathbb{P}$ and let $\alpha<\beta<\omega_{2}$. Then there is $q \leq p$ such that $(\alpha, \beta) \in \operatorname{dom}\left(c_{q}\right)$.

Proof. We may as well assume that $(\alpha, \beta) \notin \operatorname{dom}\left(c_{p}\right)$. We will define $q$ by setting $\mathcal{F}_{q}=\mathcal{F}_{p}, \operatorname{dom}\left(c_{q}\right)=\operatorname{dom}\left(c_{p}\right) \cup\{(\alpha, \beta)\}, c_{q}\left\lceil\operatorname{dom}\left(c_{p}\right)=c_{p}\right.$, and $c_{q}(\alpha, \beta)=\gamma$ for a suitably chosen $\gamma<\omega_{1}$.

The constraints on our choice of $\gamma$ are:

- $\gamma \geq f_{\beta}(\alpha)$.

- There do not exist $\alpha_{0}<\alpha_{1}<\beta$ such that $\left(\alpha_{0}, \beta\right),\left(\alpha_{1}, \beta\right) \in \operatorname{dom}\left(c_{p}\right)$ and $c_{p}\left(\alpha_{1}, \beta\right)=c_{p}\left(\alpha_{2}, \beta\right)=\gamma$.

- For every $M \in \mathcal{F}_{p}$, if $(\alpha, \beta) \in M$ then $\gamma \in M$.

Let $\delta$ be least such that there is $M \in \mathcal{F}$ with $(\alpha, \beta) \in M$ and $\delta_{M}=\delta$, if such an $M$ exists, otherwise let $\delta=\omega_{1}$. Then $f_{\beta}(\alpha)<\delta$, because the models in $\mathcal{F}$ are elementary substructures of $\left(H_{\omega_{2}}, \in,<\right)$.

We now choose $\gamma<\delta$ such that $\gamma \geq f_{\beta}(\alpha)$, and $\gamma \notin$ range $\left(c_{p}\right)$. This satisfies all the constraints.

If $G$ is $V$-generic for $\mathbb{P}$, then we define $f=\bigcup\{c: \exists \mathcal{F}(c, \mathcal{F}) \in G\}$. Lemma 9 implies that the generic function $f$ is a total function from $\left[\omega_{2}\right]^{2}$ to $\omega_{1}$. For our purposes we will need to produce ordinals $\alpha_{0}<\alpha_{1}<\beta$ such that $f\left(\alpha_{0}, \beta\right)=$ $f\left(\alpha_{1}, \beta\right)$ inside any subset of $\omega_{2}$ with order type $\omega_{1}$ in the generic extension. The following remark illustrates a potential problem:

Remark 1. Let $p=\left(c_{p}, \mathcal{F}_{p}\right)$ and let $\alpha_{0}<\alpha_{1}<\beta<\omega_{2}$ be ordinals such that $\left(\alpha_{0}, \beta\right)$ and $\left(\alpha_{1}, \beta\right)$ are not in $\operatorname{dom}\left(c_{p}\right)$. There may not exist any condition $q=\left(c_{q}, \mathcal{F}_{q}\right) \leq p$ such that $c_{q}\left(\alpha_{0}, \beta\right)=c_{q}\left(\alpha_{1}, \beta\right)$.

The issue is that potentially there is a structure $M \in \mathcal{F}_{p}$ such that $\beta \in M$, $\alpha_{0} \in M, \alpha_{1} \notin M$ and $f_{\beta}\left(\alpha_{1}\right) \geq$ delta $_{M}$. We are required by the definition of extension to have $c_{q}\left(\alpha_{0}, \beta\right) \in M$, but the definition of conditionhood implies that $c_{q}\left(\alpha_{1}, \beta\right) \geq f_{\beta}\left(\alpha_{1}\right) \geq \delta_{M}$, so that we cannot have $c_{q}\left(\alpha_{0}, \beta\right)=c_{q}\left(\alpha_{1}, \beta\right)$.

The issue isolated in Remark 1 turns out to be the only obstacle.

Lemma 10. Let $p=\left(c_{p}, \mathcal{F}_{p}\right)$ and let $\alpha_{0}<\alpha_{1}<\beta<\omega_{2}$ be ordinals such that

(1) $\left(\alpha_{0}, \beta\right)$ and $\left(\alpha_{1}, \beta\right)$ are not in $\operatorname{dom}\left(c_{p}\right)$.

(2) For every structure $M \in \mathcal{F}_{p},\left(\alpha_{0}, \beta\right) \in M \Longleftrightarrow\left(\alpha_{1}, \beta\right) \in M$.

Then there is a condition $q=\left(c_{q}, \mathcal{F}_{q}\right)$ such that $q \leq p$ and $c_{q}\left(\alpha_{0}, \beta\right)=c_{q}\left(\alpha_{1}, \beta\right)$. 
Proof. We let $\operatorname{dom}\left(c_{q}\right)=\operatorname{dom}\left(c_{p}\right) \cup\left\{\left(\alpha_{0}, \beta\right),\left(\alpha_{1}, \beta\right)\right\}$ and then set $c_{q}\left\lceil\operatorname{dom}\left(c_{p}\right)=\right.$ $c_{p}, c_{q}\left(\alpha_{0}, \beta\right)=c_{q}\left(\alpha_{1}, \beta\right)=\gamma$ for a suitable choice of $\gamma<\omega_{1}$. As in Lemma 9 we need to choose a value of $\gamma$ so that if $q=\left(c_{q}, \mathcal{F}_{p}\right)$ then $q$ is a condition, and $q$ extends $p$.

Let $\delta$ be minimal such that $\delta=\delta_{M}$ for some $M \in \mathcal{F}$ with $\left(\alpha_{0}, \beta\right) \in M$, or let $\delta=\omega_{1}$ when no such $M$ exists. By the choice of $\delta$ and the hypothesis, $\max \left\{f_{\beta}\left(\alpha_{0}\right), f_{\beta}\left(\alpha_{1}\right)\right\}<\delta$. It is now easy to check that any $\gamma$ such that $\gamma \notin \operatorname{range}\left(c_{p}\right)$ and $\max \left\{f_{\beta}\left(\alpha_{0}\right), f_{\beta}\left(\alpha_{1}\right)\right\}<\gamma<\delta$ works.

Lemma 11. $\mathbb{P}$ has the $\aleph_{2}$-chain condition.

Proof. Let $\left\langle p_{i}: i<\omega_{2}\right\rangle$ be a sequence of conditions, and let $p_{i}=\left(c_{i}, \mathcal{F}_{i}\right)$ for $i<\omega_{2}$. Let $r_{i}$ be the finite set of ordinals less than $\omega_{2}$ which appear in some element of $\operatorname{dom}\left(c_{i}\right)$, let $s_{i}$ be the countable set $\omega_{2} \cap \bigcup \mathcal{F}_{i}$, and let $x_{i}$ be the type of $\mathcal{F}_{i}$. By standard arguments using $\mathrm{CH}$ and the $\Delta$-system lemma, we may find an unbounded set $A \subseteq \omega_{2}$ and $d, C, x$ such that:

(1) $\left\langle r_{i} \cup s_{i}: i \in A\right\rangle$ forms a head-tail-tail $\Delta$-system with root $C$.

(2) For every $i \in A, c_{i} \uparrow[C]^{2}=d$.

(3) For every $i \in A$ the type of $\mathcal{F}_{i}$ is $x$.

Let $i<j$ with $i, j \in A$. Let $c=c_{i} \cup c_{j}$ and $\mathcal{F}=\mathcal{F}_{i} \cup \mathcal{F}_{j}$. We claim that $(c, \mathcal{F})$ is a condition which extends both $\left(c_{i}, \mathcal{F}_{i}\right)$ and $\left(c_{j}, \mathcal{F}_{j}\right)$. It is easy to see that $c$ is a partial function, because $c_{i}(\alpha, \beta)=d(\alpha, \beta)=c_{j}(\alpha, \beta)$ for all pairs $(\alpha, \beta) \in \operatorname{dom}\left(c_{i}\right) \cap \operatorname{dom}\left(c_{j}\right)$. It is also immediate that $c(\alpha, \beta) \geq f_{\beta}(\alpha)$ for all $(\alpha, \beta) \in \operatorname{dom}(c)$.

We claim that there do not exist ordinals $\alpha_{0}<\alpha_{1}<\alpha_{2}<\beta$ such that $c\left(\alpha_{0}, \beta\right)=$ $c\left(\alpha_{1}, \beta\right)=c\left(\alpha_{2}, \beta\right)$. Suppose for a contradiction that such ordinals exist. Since both $\left(c_{i}, \mathcal{F}_{i}\right)$ and $\left(c_{j}, \mathcal{F}_{j}\right)$ are conditions, the set of pairs of the form $\left(\alpha_{n}, \beta\right)$ must contain elements from both $\operatorname{dom}\left(c_{i}\right)$ and $\operatorname{dom}\left(c_{j}\right)$. In particular $\beta \in r_{i} \cap r_{j} \subseteq C$. Since the sets $\left\langle r_{i} \cup s_{i}: i \in A\right\rangle$ form a head-tail-tail $\Delta$-system, all the ordinals $\alpha_{0}, \alpha_{1}, \alpha_{2}, \beta$ are in $C$. Since $c_{i}\left\lceil[C]^{2}=d=c_{j}\left\lceil[C]^{2}, c_{i}\left(\alpha_{0}, \beta\right)=c_{i}\left(\alpha_{1}, \beta\right)=c_{i}\left(\alpha_{2}, \beta\right)\right.\right.$, which is a contradiction since $\left(c_{i}, \mathcal{F}_{i}\right)$ is a condition.

By the Amalgamation Lemma, $\mathcal{F}$ is a nicely arranged set with type $x$. It follows that $(c, \mathcal{F})$ is a condition in $\mathbb{P}$. It remains to show that $(c, \mathcal{F})$ is a common extension of $\left(c_{i}, \mathcal{F}_{i}\right)$ and $\left(c_{j}, \mathcal{F}_{j}\right)$, for which it will clearly suffice to show that it extends $\left(c_{i}, \mathcal{F}_{i}\right)$. By definition $c \supseteq c_{i}$ and $\mathcal{F} \supseteq \mathcal{F}_{i}$. Let $M \in \mathcal{F}_{i}$ and $(\alpha, \beta) \in \operatorname{dom}(c) \backslash \operatorname{dom}\left(c_{i}\right)$, so that $(\alpha, \beta) \in \operatorname{dom}\left(c_{j}\right)$. If $(\alpha, \beta) \in M$ then $\alpha, \beta \in r_{j} \cap s_{i} \subseteq C$; but then since $c_{i}\left\lceil[C]^{2}=d=c_{j}\left\lceil[C]^{2}\right.\right.$ we would have $(\alpha, \beta) \in \operatorname{dom}\left(c_{i}\right)$, and we conclude that $(\alpha, \beta) \notin M$. So $(c, \mathcal{F})$ extends $\left(c_{i}, \mathcal{F}_{i}\right)$.

The following lemma is the heart of this paper. It is a technical statement about conditions in $\mathbb{P}$ and countable elementary substructures which immediately implies that $\mathbb{P}$ is proper, and which will be used again in the proof that $\mathbb{P}$ adds a counterexample to $\omega_{2} \rightarrow^{\text {poly }}\left(\omega_{1}\right)_{2-b d d}^{2}$.

Before stating Lemma 12, we give some motivating remarks. To establish properness, we must show that for some large $\theta$ the following holds: for every countable elementary $N \prec H_{\theta}$ and every $p \in \mathbb{P} \cap N$, there is $p^{+} \leq p$ such that $p^{+}$is $(N, \mathbb{P})$ generic. This means that for every dense $D \in N, D \cap N$ is predense below $p^{+}$.

As usual in the context of forcing with models as side conditions, we will define $p^{+}$by adding the model $N \cap H_{\omega_{2}}$ to the family of models appearing in $p$. We must show that if $D \in N$ is dense, every $q \leq p^{+}$is compatible with some element 
$q_{1} \in D \cap N$. We will do this in a standard way; we will define a condition $q_{0} \in N$ which "saves" some information from $q$, use elementarity to find $q_{1} \in D \cap N$ with $q_{1} \leq q_{0}$, and then argue that $q_{1}$ is compatible with $q$. The distinctive feature in the statement of Lemma 12 is that after we define $q_{0}$ from $q$ and extend $q_{0}$ to $q_{1}$, we form an extension $q^{\prime}$ of $q$ with some special properties and then argue that $q^{\prime}$ is compatible with $q_{1}$; this is not necessary for the proof of properness, but is vital in the proof that the generic colouring $f$ has property $\mathrm{F} 3$ (see the proof of Lemma 13).

Before beginning the proof of Lemma 12, we recall that earlier we fixed a wellordering < of $H_{\omega_{2}}$ and defined $\mathbb{P}$ using "side conditions" built from the class $\mathcal{C}$ of countable elementary submodels of $\left(H_{\omega_{2}}, \in,<\right)$. Observe that if $\theta$ is a large regular cardinal, $<_{\theta}$ is a well-ordering of $H_{\theta}$, and $\left(H_{\omega_{2}}, \in,<\right) \in N \prec\left(H_{\theta}, \epsilon,<_{\theta}\right)$ for some countable $N$, then $N \cap H_{\omega_{2}} \in \mathcal{C}$.

Lemma 12. Let $\theta$ be a regular cardinal with $\theta>\omega_{2}$, and let $p=(c, \mathcal{F}) \in N \prec$ $\left(H_{\theta}, \in,<_{\theta}\right)$ where $<_{\theta}$ is a well-ordering of $H_{\theta}, N$ is countable, and $\left(H_{\omega_{2}}, \in,<\right) \in N$. Let $M=N \cap H_{\omega_{2}}$, and let $p^{+}=(c, \mathcal{F} \cup\{M\})$. Then

(1) $p^{+} \in \mathbb{P}$, and $p^{+} \leq p$.

(2) Let $q=(d, \mathcal{G}) \leq p^{+}$. Let $d_{0}=d \uparrow(\operatorname{dom}(d) \cap M)$, let $\mathcal{G}_{0}=M \cap \mathcal{G}$, and let $q_{0}=\left(d_{0}, \mathcal{G}_{0}\right)$. Then

(a) $q_{0} \in M \cap \mathbb{P}$.

(b) Let $q^{\prime}=\left(d^{\prime}, \mathcal{G}\right) \in \mathbb{P}$ be such that $q^{\prime} \leq q$ and $d^{\prime} \uparrow\left(\operatorname{dom}\left(d^{\prime}\right) \cap M\right)=d_{0}$, and let $q_{1} \in \mathbb{P} \cap M$ be such that $q_{1} \leq q_{0}$. Then $q^{\prime}$ and $q_{1}$ are compatible.

(3) $p^{+}$is an $(N, \mathbb{P})$-generic condition.

Proof. We break up the proof into a series of claims.

Claim 1: $p \in M$.

Proof of Claim 1. Since $\mathbb{P} \subseteq H_{\omega_{2}}, \mathbb{P} \cap N=\mathbb{P} \cap M$, in particular $p \in M$.

Claim 2: $p^{+} \in \mathbb{P}$, and $p^{+} \leq p$.

Proof. For all $L \in \mathcal{F}, L \in M$ so that $\delta_{L}<\delta_{M}$. It follows easily that $\mathcal{F} \cup\{M\}$ is a nicely arranged family, so that $p^{+}$is a condition. It is immediate from the definition that $p^{+} \leq p$.

Claim 3. $q_{0} \in M \cap \mathbb{P}$.

Proof. Since $p \in M, c \in M$ and in particular $\operatorname{dom}(c) \subseteq M$. The definition of extension in $\mathbb{P}$ implies that for every $(\alpha, \beta) \in M \cap(\operatorname{dom}(d) \backslash \operatorname{dom}(c)), d(\alpha, \beta) \in M$. So $d_{0}$ is a finite partial function from $M$ to $M$, and thus $d_{0} \in M$. Since $\mathcal{G}_{0}=M \cap \mathcal{G}$, $\mathcal{G}_{0} \in M$ and $\mathcal{G}_{0}$ is nicely arranged by Lemma 5 .

Let $q_{1}=\left(d_{1}, \mathcal{G}_{1}\right)$ be a condition in $M$ with $q_{1} \leq q_{0}$, so that $d_{1} \in M$ and $M \cap \mathcal{G}=\mathcal{G}_{0} \subseteq \mathcal{G}_{1} \subseteq M$. Now let $e=d^{\prime} \cup d_{1}$, let $\mathcal{H}$ be the diffusion of $\mathcal{G}_{1}$ from $M$ in $\mathcal{G}$, and let $r=(e, \mathcal{H})$.

Claim 4: $r$ is a condition.

Proof. By the Diffusion Lemma, $\mathcal{H}$ is nicely arranged. We claim first that $e$ is a partial function. Let $(\alpha, \beta) \in \operatorname{dom}\left(d^{\prime}\right) \cap \operatorname{dom}\left(d_{1}\right)$. Since $d_{1} \in M,(\alpha, \beta) \in$ $M \cap \operatorname{dom}\left(d^{\prime}\right)$, so that $(\alpha, \beta) \in \operatorname{dom}\left(d_{0}\right)$. Since $d_{1}$ extends $d_{0}$ and $d_{0}$ is the restriction of $d^{\prime}$ to $M, d^{\prime}(\alpha, \beta)=d_{0}(\alpha, \beta)=d_{1}(\alpha, \beta)$. 
Since $q$ and $q_{1}$ are both conditions, $e(\alpha, \beta) \geq f_{\beta}(\alpha)$ for all $(\alpha, \beta) \in \operatorname{dom}(e)$. To finish the proof that $r$ is a condition, suppose for a contradiction that $\alpha_{0}<\alpha_{1}<$ $\alpha_{2}<\beta$ and $e\left(\alpha_{0}, \beta\right)=e\left(\alpha_{1}, \beta\right)=e\left(\alpha_{2}, \beta\right)=\gamma$ for some $\gamma<\omega_{1}$. Since $q$ and $q_{1}$ are both conditions, at least one of the pairs $\left(\alpha_{i}, \beta\right)$ is in $\operatorname{dom}\left(d_{1}\right)$; it follows that $\beta \in M$ and also that $\gamma \in M$. What is more $f_{\beta}\left(\alpha_{i}\right) \leq \gamma$ for each $i$, so that $f_{\beta}\left(\alpha_{i}\right) \in M$ and so (since $f_{\beta} \in M$ and $f_{\beta}$ is injective) that $\alpha_{i} \in M$. But then all the pairs $\left(\alpha_{i}, \beta\right)$ are in $\operatorname{dom}\left(d_{1}\right)$, which is a contradiction as $q_{1}$ is a condition.

Claim 5: $r \leq q^{\prime}$.

Proof. By definition $e$ extends $d^{\prime}$ and $\mathcal{H}$ extends $\mathcal{G}$, so we need only to check that $e(\alpha, \beta) \in J$ for all $J \in \mathcal{G}$ and all $(\alpha, \beta) \in J \cap\left(\operatorname{dom}(e) \backslash \operatorname{dom}\left(d^{\prime}\right)\right)$. Now $\operatorname{dom}(e) \backslash$ $\operatorname{dom}\left(d^{\prime}\right)=\operatorname{dom}\left(d_{1}\right) \backslash \operatorname{dom}\left(d_{0}\right)$, in particular $e(\alpha, \beta)=d_{1}(\alpha, \beta)$ and $\{\alpha, \beta, e(\alpha, \beta)\} \subseteq$ $M$.

Since $e(\alpha, \beta) \in M$, we may assume without loss of generality that $\delta_{J}<\delta_{M}$. Since $\mathcal{G}$ is nicely arranged, there is $M^{\prime} \in \mathcal{G}$ such that $M^{\prime} \simeq M$ and $J \in M^{\prime}$. Let $J^{\prime}=\pi_{M^{\prime} M}(J)$ so that $J^{\prime} \in M \cap \mathcal{G}$. Since $M \cap \mathcal{G}=\mathcal{G}_{0} \subseteq \mathcal{G}_{1}, J^{\prime} \in \mathcal{G}_{1}$. Also $(\alpha, \beta) \in M$ and $(\alpha, \beta) \in J^{\prime} \subseteq M^{\prime}$, so $(\alpha, \beta)$ is fixed by $\pi_{M^{\prime} M}$ and hence $(\alpha, \beta) \in J^{\prime}$. Since $q_{1}=\left(d_{1}, \mathcal{G}_{1}\right) \leq q_{0}=\left(d_{0}, \mathcal{G}_{0}\right)$, and $J^{\prime} \in \mathcal{G}_{0}$, it follows from the definition of extension that $d_{1}(\alpha, \beta) \in J^{\prime}$. Since $e(\alpha, \beta)=d_{1}(\alpha, \beta)$ and $\delta_{J}=\delta_{J^{\prime}}$, we are done.

Claim 6: $r \leq q_{1}$.

Proof. By definition $e$ extends $d_{1}$ and $\mathcal{H}$ extends $\mathcal{G}_{1}$, so we need only to check that $e(\alpha, \beta) \in J$ for all $J \in \mathcal{G}_{1}$ and all $(\alpha, \beta) \in J \cap\left(\operatorname{dom}(e) \backslash \operatorname{dom}\left(d_{1}\right)\right)$. We claim that no such $J$ and $(\alpha, \beta)$ exist. To see this observe that $J \subseteq M$ and $(\alpha, \beta) \in \operatorname{dom}\left(d^{\prime}\right)$, so that $(\alpha, \beta) \in \operatorname{dom}\left(d_{0}\right)$ contradicting the assumption that $(\alpha, \beta) \notin \operatorname{dom}\left(d_{1}\right)$.

Claim 7: $p^{+}$is $(N, P)$-generic.

Proof. Let $D \in N$ be a dense subset of $\mathbb{P}$ and let $q \leq p^{+}$. Since $q_{0} \in N$, there is $q_{1} \in D \cap N$ with $q_{1} \leq q_{0}$. Then $q_{1} \in M$, and it follows by Claims $4-6$ (for this particular choice of $q_{1}$ ) that $q$ and $q_{1}$ are compatible.

This concludes the proof of Lemma 12.

Lemma 13. Let $G$ be $\mathbb{P}$-generic over $V$, and let $f:\left[\omega_{2}\right]^{2} \rightarrow \omega_{1}$ be the corresponding generic function. Let $X$ be a set in $V[G]$ with $X \subseteq \omega_{2}$ and $\operatorname{ot}(X)=\omega_{1}$. Then there exist ordinals $\alpha_{0}, \alpha_{1}, \beta \in X$ such that $\alpha_{0}<\alpha_{1}<\beta$ and $f\left(\alpha_{0}, \beta\right)=f\left(\alpha_{1}, \beta\right)$.

Proof. Let $\dot{X}$ be a name for such a set of ordinals, and let $p=(c, \mathcal{F}) \in \mathbb{P}$ and $\gamma<\omega_{2}$ be such that $p \Vdash \sup (\dot{X})=\gamma$. Fix some large regular $\theta$ and find a countable elementary submodel $N \prec\left(H_{\theta}, \in,<_{\theta}\right)$ such that $\left\{p, \mathbb{P}, \dot{X},\left(H_{\omega_{2}}, \in,<\right)\right\} \subseteq N$. Let $M=N \cap H_{\omega_{2}}$ and $p^{+}=(c, \mathcal{F} \cup\{M\})$.

Since $c f(\gamma)=\omega_{1}, N \cap \gamma$ is bounded in $\gamma$. Find $q=(d, \mathcal{G}) \leq p^{+}$and $\beta$ such that $\sup (N \cap \gamma)<\beta<\gamma$, and $q \Vdash \beta \in \dot{X}$. Let $d_{0}=d \uparrow(\operatorname{dom}(d) \cap M)$, and $\mathcal{G}_{0}=M \cap \mathcal{G}$, so that by Lemma $12 q_{0}=\left(d_{0}, \mathcal{G}_{0}\right) \in M \cap \mathbb{P}$.

By elementarity we may find $q_{1} \leq q_{0}$ such that $q_{1} \in N \cap \mathbb{P}$ (which equals $M \cap \mathbb{P}$ ) and ordinals $\alpha_{i} \in N \cap \gamma$ for $i<2^{|\mathcal{G}|}+1$ such that

- $\left(\alpha_{i}, \beta\right) \notin \operatorname{dom}(d)$.

- $i<j \Longrightarrow \alpha_{i}<\alpha_{j}$. 
- For each $i, q_{1} \Vdash \alpha_{i} \in \dot{X}$.

By the pigeonhole principle there are $i, j$ with $i<j$ such that for every $J \in \mathcal{G}$, $\left(\alpha_{i}, \beta\right) \in J \Longleftrightarrow\left(\alpha_{j}, \beta\right) \in J$. By Lemma 10 there is $q^{\prime}=\left(d^{\prime}, \mathcal{G}\right) \leq q$ such that $\operatorname{dom}\left(d^{\prime}\right)=\operatorname{dom}(d) \cup\left\{\left(\alpha_{i}, \beta\right),\left(\alpha_{j}, \beta\right)\right\}$ and $d^{\prime}\left(\alpha_{i}, \beta\right)=d^{\prime}\left(\alpha_{j}, \beta\right)$.

The hypotheses of Lemma 12 are satisfied and so there is a condition $r$ which is a common extension of $q^{\prime}$ and $q_{1}$. Clearly $r \Vdash\left\{\alpha_{i}, \alpha_{j}, \beta\right\} \subseteq \dot{X}$ and $r \Vdash \dot{f}\left(\alpha_{i}, \beta\right)=$ $\dot{f}\left(\alpha_{j}, \beta\right)$.

We have proved:

Theorem 2. Let $C H$ hold. Then there is a proper $\aleph_{2}$-c.c. forcing poset such that the negative polychromatic relation $\omega_{2} \nrightarrow^{\text {poly }}\left(\omega_{1}\right)_{2-b d d}^{2}$ holds in the generic extension.

\section{OpEN PROBLEMS}

We conclude by discussing some open problems about polychromatic Ramsey theory at small cardinals. As we already mentioned in the introduction, the situation for partitions of pairs at $\omega_{1}$ is reasonably well understood: $\omega_{1} \rightarrow^{\text {poly }}(\alpha)_{k-b d d}^{2}$ for $k<\omega$ and $\alpha<\omega_{1}$, under CH $\omega_{1} \nrightarrow^{\text {poly }}\left(\omega_{1}\right)_{2-b d d}^{2}$, by contrast PFA implies that $\omega_{1} \rightarrow^{\text {poly }}\left(\omega_{1}\right)_{<\omega \text {-bdd }}^{2}$, and finally MA does not suffice to imply this positive relation.

However even at $\omega_{1}$ the situation for partitions of triples remains mysterious. The ordinary partition relation $\omega_{1} \rightarrow(\omega+2)_{2}^{3}$ is known to be false in ZFC, so Galvin's result G1 from the introduction is of limited help.

Problem 1: What is the extent of the partition relation $\omega_{1} \rightarrow^{\text {poly }}(\alpha)_{2-b d d}^{3}$ ? In particular is it consistent that $\omega_{1} \rightarrow^{\text {poly }}\left(\omega_{1}\right)_{2-b d d}^{3}$ ?

Turning to $\omega_{2}$, one very natural question is whether there is any analogue of Todorčević's PFA result for $\omega_{1}$. We are very skeptical that the following question has a positive answer but see no way to construct a counterexample.

Problem 2: Is it consistent that $\omega_{2} \rightarrow^{\text {poly }}\left(\omega_{2}\right)_{2}^{2}$ bdd ?

Moving to larger cardinals, we recall that under GCH $\kappa^{+} \rightarrow^{\text {poly }}(\eta)_{<\kappa-\text { bdd }}^{2}$ for every regular $\kappa$ and every $\eta<\kappa^{+}$. The proof appears to break down for $\kappa$ singular, and experience with singular cardinals suggests that there may be an essential difficulty here.

Problem 3: Does GCH imply that $\kappa^{+} \rightarrow^{\text {poly }}(\eta)_{<\kappa \text {-bdd }}^{2}$ for every $\eta<\kappa^{+}$when $\kappa$ is singular? If not, does GCH plus $\square_{\kappa}$ imply this?

Returning finally to $\omega_{2}$ and problems more directly related to this paper, we note that the model of Theorem 2 is obtained by a "one shot" forcing extension in which $\omega_{2}$ reals are added to a model of $\mathrm{CH}$ by a proper $\omega_{2}$-c.c forcing poset. This limits our control over the final model. In particular we ask:

Problem 4: Is it consistent with MA that $\omega_{2} \nrightarrow^{\text {poly }}\left(\omega_{1}\right)_{2-b d d}^{2}$ ?

Problem 5: Is it consistent with $2^{\omega}>\omega_{2}$ that $\omega_{2} \nrightarrow^{\text {poly }}\left(\omega_{1}\right)_{2-b d d}^{2}$ ?

Acknowledgement: We would like to thank the anonymous referee for their very detailed and constructive comments on the first version of this article. 


\section{REFERENCES}

[1] U. Abraham, J. Cummings, and C. Smyth, Some results in polychromatic Ramsey theory, Journal of Symbolic Logic 72 (2007), 865-896.

[2] J. Baumgartner and A. Hajnal, A proof (involving Martin's axiom) of a partition relation, Fundamenta Mathematicae 78 (1973), 193-203.

[3] F. Galvin, letters to S. Todorčević, cited in [7].

[4] F. Galvin, Advanced Problem number 6034, American Mathematical Monthly, 82 (1975), 529.

[5] P. Koszmider, On strong chains of uncountable functions, Israel Journal of Mathematics 118 (2000), 289-315.

[6] S. Shelah and J. Zapletal, Embeddings of Cohen algebras, Advances in Mathematics 126 (1997), 93-118.

[7] S. Todorčević, Forcing positive partition relations, Transactions of the American Mathematical Society 280 (1983), 703-720.

[8] S.Todorčević, A note on the proper forcing axiom, in Axiomatic set theory, Contemporary Mathematics 31, American Mathematical Society, Providence, RI, 1984.

[9] S. Todorčević, Directed sets and cofinal types, Transactions of the American Mathematical Society 290 (1985), 711-723.

Mathematics department and computer science Department, Ben-Gurion University, BeER-SHeva, 84105 IsRAel

Department of Mathematical Sciences, Carnegie Mellon University, Pittsburgh PA 15213, USA 